\title{
23
}

\section{Weighting Spatial Relationships in Retrieval by Visual Contents}

\author{
A.Del Bimbo, and E.Vicario \\ Dipartimento Sistemi e Informatica, Università di Firenze \\ 3, via santa Marta, 50139 Firenze, Italy, \\ \{delbimbo,vicario\}@aguirre.ing.unifi.it
}

\begin{abstract}
An original framework is presented which supports representation and visual querying of images based on spatial arrangements of typed objects. Design assumptions taken in the design of the framework are motivated with reference to the concrete case of retrieval by visual contents within a library of digital images reproducing renaissance paintings. Properties of the framework are expounded to develope an efficient computation technique and to motivate and assess a metric of similarity for quantitative comparison of spatial relationships between region pairs. Representation and comparison of binary relationships betwen regions is then embedded within a graph-theoretical framework supporting representation and comparison of the spatial arrangements of images.
\end{abstract}

\section{Keywords}

Image Databases, Visual Indexing, Query by Visual Contents, Visual Query Languages, Spatial Relationships, Approximate Matching

\section{INTRODUCTION}

With the recent advances in multimedia technology, a growing attention is being focused on libraries of digital images. Effective access to such archives requires that conventional searching techniques based on external attributes of the image, such as pressmarks, authorship or historical keywords, are complemented by content-based queries addressing appearing visual features of searched data. Central to this retrieval approach is the creation of models, which permit to abstract images into some space of features and support indexing and comparison of visual contents. Depending on the specific characteristics of the images at hand, such models can rely on different facets of the informative contents of visual data, such as the color and texture distribution, the shape of appearing objects, and their spatial relationships [12] [10] [6]. 
In general, content modeling must follow the analysis of the salient features which mainly characterize contents and regularities in the specific images at hand. This analysis permits to shape expressivity of user queries and drives the solution of a number of tradeoffs related with such issues as semantic level of queries, automatic/manual description of contents, visual interaction paradigm in the formulation of queries. In this paper, we focus on visual modeling of images reproducing paintings of the italian renaissance period. This type of paintings constitute a relevant portion of the italian cultural heritage, and directly affects a number of applications for inventorying and analysis and of cultural goods, multimedia authoring, education.

\subsection{Renaissance Paintings: the Engineering Perspective}

Contents of renaissance paintings are largely pervaded by cultural and iconographic conventions, which reflect historical evolution of painting practice as well as artistic effort to drive interpretation of represented episodes. A large part of these conventions are encoded in the visual appearance of individual characters and in their spatial arrangement. This results in a number of regularities which can be translated into design assumptions for the development of a content modeling approach based on spatial relationships among typed objects.

Objects appearing within renaissance paintings can be easily distinguished in a limited number of dominating characters and a set of background and secondary elements. Both types of objects are produced by the explicit anthor's intention rather than by accidental facts, as may happen within photographs taken from reality. Dominating objects are usually less than ten, and background components can be naturally grouped in collective terms such as landscapes, or person groups. In the large majority of cases, ten to thirty objects are sufficient to provide a detailed description of the contents of a painting. This largely motivates the assumption of human-assisted methods in the recognition of imaged objects. The cost of this recognition is marginal with respect to the time spent in carrying out conventional textual classification.

Object types recur through different paintings of different authors, and their appearance and spatial composition is largely driven by iconographic conventions related with the reproduced episode. A vocabulary of a few tens of types encompasses most of the characters across the majority of cases, while exhaustive description can be accomplished with a few hundreds of types.

Relevant aspects of the mutual positioning of objects are captured by directional relationships such as above, below, left of. Only a minor relevance is associated with topological adjacency, which often depends on accidental occlusions in the $2 \mathrm{D}$ representation of a $3 \mathrm{D}$ scene.

Spatial relationships are usually vague and tend to evade a single crisp 
description. It is often the case that the relationship between two objects is correctly described by contrasting statements with different degrees of truth. Much of this vagueness arises from the fact that objects are curvilinear and complex in shape, and that their distances are small with respect to their dimensions. This basically prevents assimilation of objects to their minimum embedding rectangles as well as to their punctual centroids.

Imaginary scenes reproduced in the paintings are usually taken from a point of view, which follows the canon of observer's frontality, and which is strictly related with iconographic conventions about the represented episode. This enables description of the spatial arrangement of imaged objects with respect to the reference system of the overall painting. Due to the use of mathematical perspective, also enforced by the presence of architectural geonietrical elements such as textured floors, columns rows, and walls, contents of the 3D reproduced scene can be completely represented in terms of $2 \mathrm{D}$ relationships appearing in the painting. In particular, foreground/background relationships are implicitly encoded in the mutual appearing dimension of objects.

\subsection{Modeling Spatial Relationships Between Typed Objects}

Spatial-based modeling addresses the relationships among imaged pixel regions. These are expressed in terms of set-theoretical concepts such as inclusion, overlapping or adjacency [7] [13], or in terms of directional constructs such as left of, above, below, and so on [3] [9] [11]. Most of the the work in spatial modeling for image databases develops on the theory of the symbolic projection [3] [4], which reduces both directional and topological relationships to the linear ordering of objects projections on two reference axes. This reduces matching from quadratic to linear complexity, under the assumption that objects are replaced through a single representative point, usually taken in the centroid. A large work has been done around the model to account objects extent, trading matching efficiency for the sake of representation soundness.

In a first approach, initiated by the $2 D G$-string and perfected with the 2DC-string, objects are cut in subparts with disjoint convex hulls [2][15]. This permits to maintain a sequential representation of the overall ordering, but may result in complex segmentation, as the cuts performed on each objects depend on the total number of objects in the scene. This yields quadratic complexity, which basically counterbalances the actual advantage deriving from the usage of a sequential representation.

In a second approach, in the $2 D-B$ string [16] [14], the ordering of objects is represented in terms of the ordering of the starting and ending points of the intervals obtained by projecting objects on the reference axes. This basically leads to a sort of 2D extension of the Allen's interval logic [1], with 13 exclusive ordering conditions on each axis resulting in $13 \times 13$ mutually exclu- 
sive cases in the relationships between any two objects. Since projections on different axes are independent, the representation subtends the assimilation of objects to their minimum embedding rectangles, which may be unsufficient to discriminate perceptually distant arrangements.

In [5], this limitation is partially overcome by replacing each object through a finite set of representative points, and by representing the relationship between an observing and an observed object as a set of walkthroughs capturing the ordering conditions among representative points of the two objects. By permitting a spatial relationship to be qualified as a set of different, and possibly contrasting, ordering conditions, this representation attempts to match the native vagueness of human perception of spatial arrangements of finite and curvilinear objects.

An inherent limitation of the theory of the symbolic projection in the context of image databases is its symbolic nature itself, which inherently subtends Boolean classification of spatial arrangements into a set of mutually exclusive cases. While facilitating indexing and logical inference, the lack for numeric values results in classification thresholds, which prevent a continuous relationship between perceived spatial arrangements and their representations. This largely hurdles the institution of quantitative metrics of similarity and basically limits the robustness of comparison. To overcome this limitation, directional information must be associated with a non-symbolic orientation measure.

In [11], directional information is represented through the numeric value of the orientation of the line connecting object centroids. This permits evaluation of a finite distance between non-exactly matching arrangements, and opens the way to recognition algorithms which ensure continuity in the relation between measured and perceptual similarity. The critical condition for the applicability of the approach is the replacement of an object through a single representative point. If this point is the object centroid, the directional relationship captures a sort of average directional relationship. However, this average value may loose soundness in the presence of complex shaped objects or when object distances are small with respect to object dimensions. This may prevent distinction between perceptually dissimilar configurations.

In this paper, we introduce an original modeling technique which enables quantitative representation and comparison of the mutual positioning of a pair of extended regions, and which is able to account for the overall distribution of relationships among the individual pixels belonging to the two regions. By developing on the modeling style of walkthroughs suggested in [5], the relationship between an observing and an observed object is represented by a finite set of equivalence classes (the symbolic walkthroughs) on the dense sets of possible paths leading from any pixel in the observing object to any pixel in the observed object. Each such equivalence class is associated with a weight which provides an integral measure of the set of pixel pairs that are connected by a path belonging to the class, thus accounting for 




Figure 1 Cartesian paths from $A$ to $B$ are collected in three independent walkthroughs $\langle 1,1\rangle,\langle 1,0\rangle$, and $\langle 1,-1\rangle$.

the degree of truth by which the individual class represents the actual relationship between the two regions. Mirroring this organization, the resulting representation is referred to as weighted walktrhoughs model. Properties of weighted walkthroughs are expounded to develope an efficient computation technique and to motivate and assess a metric of similarity for quantitative comparison of spatial relationships between region pairs. Representation and comparison of binary relationships betwen regions is then embedded within a graph-theoretical framework supporting representation and comparison of the spatial arrangements of images.

\section{WEIGHTED WALKTHROUGHS}

Given a pair of objects $A$ and $B$ within a Cartesian reference system, the set of Cartesian paths from $A$ to $B$ is the set of paths leading from any point of $A$ to any point of $B$ along two subsequent horizontal and vertical steps. Walkthroughs from $A$ to $B$ are equivalence classes on the set of Cartesian paths from $A$ to $B$, which abstracts from: the two connected points of $A$ and $B$; the distance covered by each step; and the order in which the two steps are taken. For any two objects $A$ and $B$, there exist at most 9 independent walkthroughs, which can be represented by a pair of indices $\langle i, j\rangle$, taking values $-1,0$ or +1 : index $i$ is equal to -1 , 0 or +1 , whether the step along the horizontal axis has negative orientation or null length or positive orientation, respectively; in a similar manner, index $j$ encodes the step along the vertical axis. Fig.1 reports an example helping intuitive understanding.

In order to account for its perceptual relevance, each walkthrough $\langle i, j\rangle$ is associated with a weight $w_{i, j}(A, B)$, which measures the number of pairs of points belonging to $A$ and $B$ which are connected through a Cartesian path equivalent to the walkthrough $\langle i, j\rangle$ itself. This weight is evaluated 


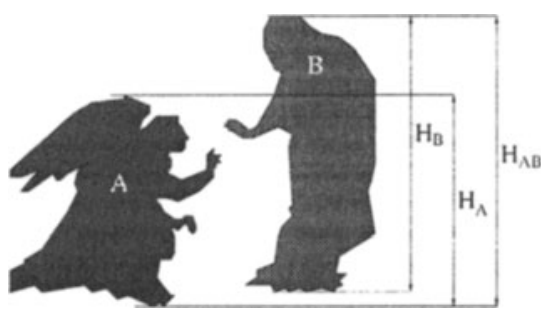

Figure 2 Meaning of symbols appearing in Eq. (2).

by taking a four-dimensional integral over the pairs of points belonging to objects $A$ and $B$ :

$w_{i, j}(A, B)=K_{i j}(A, B) \int_{A} \int_{B} C_{i}\left(x_{b}-x_{a}\right) C_{j}\left(y_{b}-y_{a}\right) d x_{b} d y_{b} d x_{a} d y_{a}$.

where:

- $C_{-1}(\cdot)$ and $C_{+1}(\cdot)$ denote the characteristic functions of negative and positive real numbers $\mathcal{R}^{-}$and $\mathcal{R}^{+}$, respectively, while $C_{0}(\cdot)$ denotes the Dirac function $\delta(\cdot)$ which here acts as characteristic function of the singleton set $\{0\}$;

- $K_{i j}(A, B)$ is a dimensional normalization factor which is defined differently whether indexes $i$ and $j$ are equal to zero or take non-null values $I$ and $J$.

$$
\begin{aligned}
K_{I, J}(A, B) & =\frac{1}{|A||B|} \\
K_{I, 0}(A, B) & =\frac{1}{H_{A B} L_{A} L_{B}} \\
K_{0, J}(A, B) & =\frac{1}{L_{A B} H_{A} H_{B}} \\
K_{0,0}(A, B) & =\frac{1}{\sqrt{|A||B|}}
\end{aligned}
$$

where: $|A|$ and $|B|$ are 2-dimensional measures of $A$, and $B ; L_{A}, H_{A}$, $L_{B}$ and $H_{B}$ are the width and the height of $A$ and $B$, respectively; $L_{A B}$, and $H_{A B}$ are the width and height of the minimum embedding rectangles of the union of $A$ and $B$. Fig.2 helps intuitive understanding.

The 9-tuple of weights $w_{i, j}(A, B)$ provides a symbolic representation of the overall position of object $B$ with respect to the object $A$. This representation abstracts from details such as the actual shapes of the objects and the quantitative distances among their individual points, and rather focuses on the joint distribution of masses within the two objects. Please note that the 
use of Dirac function in the expression of the characteristic function of the origin of real numbers ensures a finite non-null measure also for weights that are obtained by the integration of a quasi-everywhere null function. Different normalization factors $K_{i, j}$ are used to compensate the effect on dimensionality of the integrals which derives from the use of such Dirac functions: while $K_{I, J}$ is a space length to the fourth power, space dimensionalities of $K_{I, 0}$, $K_{0, J}$, and $K_{0,0}$ are reduced to the third, the third, and the second power, respectively.

\subsection{Topological Invariance and Compositionality}

The 9 coefficients in a weighted walkthrough representation are invariant with respect to shifting and zooming of the image, and they are anti-commutative with respect to the objects $A$ and $B$ to which they refer:

$$
w_{i, j}(A, B)=w_{-i,-j}(B, A)
$$

Weighted walkthroughs are compositional, i.e. for any object $A$ and for any two disjoint objects $B_{1}$ and $B_{2}$, the weight $w_{i, j}\left(A, B_{1} \cup B_{2}\right)$ can be derived by linear combination of weights $w_{i, j}\left(A, B_{1}\right)$ and $w_{i, j}\left(A, B_{2}\right)$. Specifically, for $I$ and $J$ different than 0 :

$$
\begin{aligned}
w_{I, J}(A, B) & =\frac{\left|B_{1}\right|}{|B|} w_{I, J}\left(A, B_{1}\right)+\frac{\left|B_{2}\right|}{|B|} w_{I, J}\left(A, B_{2}\right) \\
w_{I, 0}(A, B) & =\frac{H_{A, B_{1}} L_{B_{1}}}{H_{A, B} L_{B}} w_{I, 0}\left(A, B_{1}\right)+\frac{H_{A, B_{2}} L_{B_{2}}}{H_{A, B} L_{B}} w_{I, 0}\left(A, B_{2}\right) \\
w_{0, J}(A, B) & =\frac{L_{A, B_{1}} H_{B_{1}}}{L_{A, B} H_{B}} w_{0, J}\left(A, B_{1}\right)+\frac{L_{A, B_{2}} H_{B_{2}}}{L_{A, B} H_{B}} w_{0, J}\left(A, B_{2}\right) \\
w_{0,0}(A, B) & =\frac{\sqrt{\left|B_{1}\right|}}{\sqrt{|B|}} w_{0,0}\left(A, B_{1}\right)+\frac{\sqrt{\left|B_{2}\right|}}{\sqrt{|B|}} w_{0,0}\left(A, B_{2}\right)
\end{aligned}
$$

\subsection{Weighted Walkthroughs between Rectangles}

The property of compositionality of Eq.(3) permits to reduce the relationships between any two objects to a linear combination of the relationships annong the parts of any their decomposition. In particular, if objects are assimilated to multi-rectangles, the evaluation of the four-dimensional integral of Eq.(1) can be reduced to the linear composition of a set of sub-integrals taken over rectangular domains. This permits to derive the relationship between any two multi-rectangular objects $A$ and $B$ by linear composition of close-form terms computed in a strict set of independent variants of the mutual position between two rectangles. These variants are 9 and correspond to 3 basic cases. 
Case 1: Projections of the two objects are disjoint on both axes. Four variants are possible. In particular, if object $B$ is in the right-lower quadrant of $A$, we have:

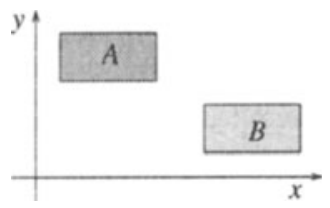

$$
w(A, B)=\left[\begin{array}{lll}
0 & 0 & 0 \\
0 & 0 & 0 \\
0 & 0 & 1
\end{array}\right]
$$

Similar results are obtained for the remaining three variants.

Case 2: Projections of objects $A$ and $B$ are completely superposed on one axis and disjoint on the other axis. Four variants of the case are possible. In particular, if objects $A$ and $B$ are aligned along the horizontal direction, we have:

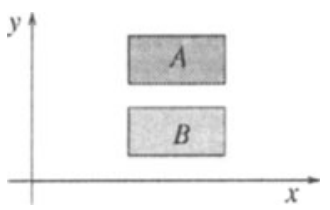

$$
w(A, B)=\left[\begin{array}{lll}
0 & 0 & 0 \\
0 & 0 & 0 \\
\frac{1}{2} & 1 & \frac{1}{2}
\end{array}\right]
$$

Of course, similar conditions hold for the variant in which the positions of objects $A$ and $B$ are inverted and for the two variants in which $t$ and $B$ are aligned along the horizontal direction.

Case 3: Projections of objects $A$ and $B$ are completely superimposed on both the axes. The case corresponds to $A$ and $B$ completely superimposed, and has a single variant:

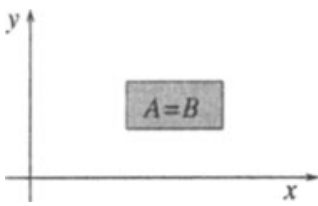

$$
w(A, B)=\left[\begin{array}{ccc}
\frac{1}{4} & \frac{1}{2} & \frac{1}{4} \\
\frac{1}{2} & 1 & \frac{1}{2} \\
\frac{1}{4} & \frac{1}{2} & \frac{1}{4}
\end{array}\right]
$$

The reduction of multi-rectangular compositions to a strict nucleus of 9 basic variants not only simplifies analytical treatment and effective implementation of spatial modeling based on walkthroughs. Rather, it also ensures consistency in quantitative weights associated with relationships which may be decomposed in different manners. 


\subsection{A Metric of Similarity for Weighted Walkthroughs}

Similarity between weighted walkthroughs can be evaluated by computing a numeric distance in the space of 9-tuples. Specifically, similarity $\mathcal{S}(w, \bar{w})$ between a pair of 9-tuples $w$ and $\bar{w}$ is evaluated as the the difference to 1 of a distance $\mathcal{D}(w, \bar{w})$ :

$\mathcal{S}(w, \bar{w})=1-\mathcal{D}(w, \bar{w})$

In turn, $\mathcal{D}(w, \bar{w})$ is evaluated as a convex normal combination of four components which evaluate the distance between homologous coefficients in corner positions, in middle horizontal and vertical positions, and in the central position of the weighted walkthrough 9-tuples $w$ and $\bar{w}$.

$\mathcal{D}(w, \bar{w})=\alpha d_{x y}(w, \bar{w})+\beta d_{x o}(w, \bar{w})+\gamma d_{o y}(w, \bar{w})+\delta d_{o o}(w, \bar{w})$

where $\alpha, \beta, \gamma$, and $\delta$ are non-negative numbers with sum equal to 1 , and $d_{x y}, d_{x o}, d_{o y}$, and $d_{o o}$ are distance components defined as follows:

$$
\begin{aligned}
& d_{x y}=\frac{1}{2} \sum_{I= \pm 1, J= \pm 1}\left|w_{I, J}-\bar{w}_{I, J}\right| \\
& d_{x o}=\frac{1}{2} \sum_{I= \pm 1}\left|w_{I, 0}-\bar{w}_{I, 0}\right| \\
& d_{o y}=\frac{1}{2} \sum_{J= \pm 1}\left|w_{0, J}-\bar{w}_{0, J}\right| \\
& d_{o}=\left|w_{0,0}-\bar{w}_{0,0}\right|
\end{aligned}
$$

Properties of distance $\mathcal{D}$ can be derived from the analysis of the range of possible values for the 9-tuples of weighted walkthroughs. These are nonnegative upper-bounded numbers.

- The sum of the four corner coefficients of the representation always evaluates to 1 .

- The sum of two opposite middle coefficients is never greater than 1 .

- The central element $w_{0,0}(A, B)$ is never greater than 1 .

By exploiting upper-bounds on weighted walkthroughs, distance $\mathcal{D}$ can be proven to exhibit the five properties that are commonly assumed as axiomatic basis of a dis-similarity measure, i.e. positivity $(\mathcal{D}(w, \bar{w}) \geq 0)$, normality $(\forall w, \bar{w}, \mathcal{D}(w, \bar{w}) \leq 1)$, autosimilarity $(\mathcal{D}(w, \bar{w})=0$ iff $w \equiv \bar{w})$, simmetry $(\mathcal{D}(w, \bar{w})=\mathcal{D}(\bar{w}, w))$, and triangularity $(\mathcal{D}(w, \bar{w})+\mathcal{D}(\bar{w}, \hat{w}) \geq \mathcal{D}(w, \hat{w}))$. 
The properties are proven by inductive extension through Eq.(3) of the properties holding in the 9 basic variants of Sect.2.2. Each property is proven to separately hold for each of the four components $d_{x y}, d_{x o}, d_{o y}$, and $d_{o o}$, and it is then extended to the sum $\mathcal{D}$ by the assumption that $\alpha, \beta, \gamma$, and $\delta$ comprise a convex and normal combination.

The five properties of positivity, normality, autosimilarity, simmetry, and triangularity provide a theoretical argument for the soundness of the metric of similarity on weighted walkthroughs. Beside this argument, the plausibity of the metric is also supported by computational experience and by the property of continuity in the relation between object arrangements and their weighted walkthrough representation. Detailed treatment of this property is not in the scope of this paper. Roughly speaking, this continuity means that slight changes in the mutual positioning or in the mass distribution of two objects $A$ and $B$ themselves, result into slight changes in the weighted walkthroughs between $A$ and $B$. Demonstration relies on the fact that changes in weighted walkthroughs are related through an integral evaluation to the spatial distribution of objects.

\section{USING WEIGHTED WALKTHROUGHS TO MODEL SPATIAL ARRANGEMENTS}

Weighted walkthroughs represent binary spatial relationships between pairs of objects. This representation can be embedded within a graph theoretical framework for the representation of spatial arrangements among set of typed objects (types allowing for multiple instances). In this section, we expound one such framework as it was designed and implemented within a prototype system supporting retrieval by contents from a digital library of images reproducing renaissance paintings.

Briefly described, the system follows a three-stage operation model distinguishing archiving, querying and retrieval. At archiving time, a concrete description is associated with each stored picture so as to retain information about the types of appearing objects and about their mutual weighted walkthroughs. In the querying stage, the abstract class of images that are to be retrieved is specified by the user by means of a sketch reproducing characterizing objects and spatial relationships. Finally, retrieval is performed by means of a model checking algorithm which compares the weighted walkthroughs of the sketch against concrete image descriptions and returns a test score measuring the resulting degree of similarity. 


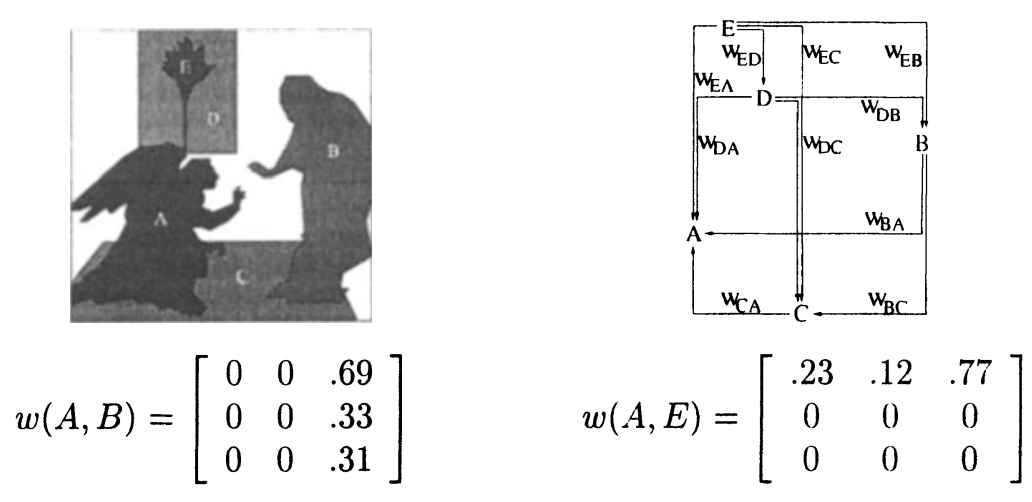

Figure 3 The sketch of an image, its representation as a graph, and weighted walkthroughs between objects $A$ and $E$, and between objects $A$ and $B$ In the graph, nodes labeled with the object type and edges are labeled with weighted walkthroughs representing the mutual relationship between connected objects.

\subsection{Image Description}

At archiving time, each image is associated with a description capturing identity and types of imaged objects along with their mutual weighted walkthroughs. One such description can be represented as a triple description $=<$ Obj,Type, $W W>$, where: $O b j$ is a set of objects; Type associates each object with a type within an infinite support Types: Type: Obj $\rightarrow$ Types : and $W W$ associates each pair of objects with a weighted walkthrough (ap)turing their mutual spatial relationship:

$$
\begin{aligned}
& W W: O b j \times O b j \rightarrow[0,1]^{9} \\
& W W\left(o b j_{1}, o b j_{2}\right)=w\left(o b j_{1}, o b j_{2}\right)
\end{aligned}
$$

It is useful to note that a description can be regarded as complete graph in which vertices are objects and edges are spatial relationships. In this perspective, each vertex is labeled with a type, and each edge is labeled with the weighted walkthrough associated with the relationship that it represents. Fig.3 shows the sketch of an image and its description in the form of labeled graph.

The construction of an image description is obtained through conventional visual interaction enabling the user to contour and classify relevant imaged objects. This effort results to be by far secondary with respect to the time spent in conceptual analysis and textual classification of contents. 

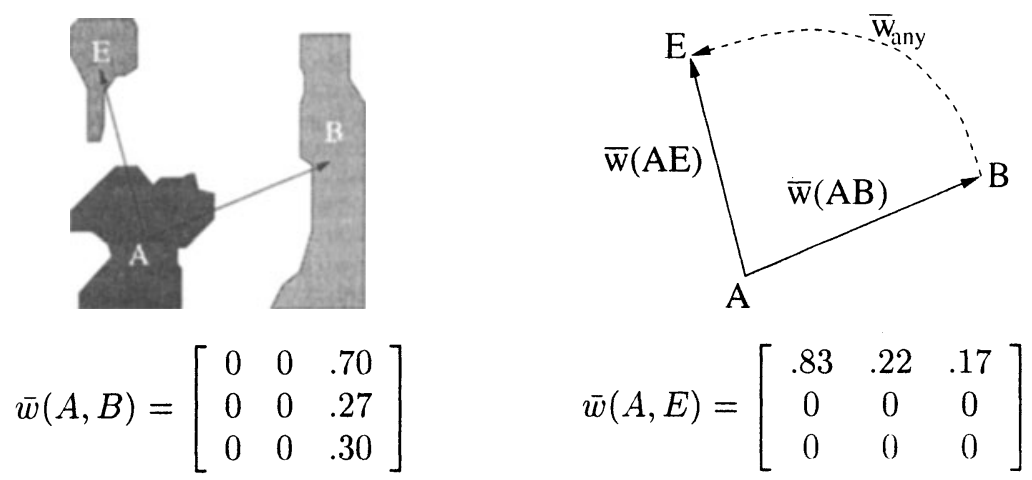

Figure 4 The sketch of a query, its representation as a graph according to the style of Fig.3, and the weighted walkthroughs between objects $A$ and $B$ and between objects $A$ and $E$. In the sketch, relationships between $A$ and $E$, and between $A$ and $B$ have been marked as relevant to the purposes of the query. In the graph, a fictitious edge, drawn as a dotted line, is added between objects $B$ and $E$ to permit application of the chinese postman algorithm. The fictitious edge is labeled with the conventional relationship $\bar{w}_{a n y}$ which is assumed to exactly match any relationship $w\left(\right.$ i.e. $\mathcal{S}\left(\bar{w}_{a n y}, \cdot\right)=1$ ).

\subsection{Query Specification}

Queries are formulated as properties of the spatial relations among typed objects in the picture. These properties are specified by-example by drawing and arranging typed icons on a composition screen. The interpretation of the arrangement can be captured within a querying graph which basically shares the same syntactic structure of the description graph used in image descriptions. The only difference is that, in the querying stage, the user is allowed to express a concept of relevance by selecting which object relationships appearing in the example are actually relevant for the query. In the syntactic perspective, this implies that the querying graph is not complete, so that the query can be formally represented as a triple query $=\langle O \bar{b} j, W \bar{W}, T \bar{y} p e>$. where: $O \bar{b} j$ is a set of nodes; Type associates each object with a type in the predefined set Types; and $\overline{W W}$ associates a subset of the pairs of objects with a weighted walkthrough:

$$
\begin{aligned}
& S i g \subseteq O \overline{O b} j \times O \overline{O b} j \\
& W \bar{W}: S i g \rightarrow[0,1]^{9} \\
& \overline{W W}\left(<o b j_{1}, o b j_{2}>\right)=\bar{w}\left(o b j_{1}, o b j_{2}\right) \text {, }
\end{aligned}
$$

Fig. 4 shows the sketch of a query and its description in the form of a noncompletely-connected labeled graph. 


\subsection{Automated Model Checking}

Model checking of a query against an image description can be formulated as a problem of weighted sub-graph matching. The problem consists in finding a subgraph of the image description which matches the object types of the query and maximizes the similarity of spatial relationships. To permit recursive management of weights, the querying graph is represented in the form of a sequential formula and then checked against the description graph by means of a recursive labeling algorithm.

To this end, the set of constraints expressed in the query are represented as a linear path which traverses all the edges of the querying graph. If each vertex of the graph has an even number of edges, the traversing path exists and can be determined in polynomial time through the so-called "algorithm of the chinese postman" [8]. Nodes with an odd number of edges can be reduced to the necessary condition for the application of the algorithm by introducing fictitious edges whose labeled walkthrough is assumed to exactly match any relationship against which they are compared.

In the case of the example of Fig.4, nodes $E$ and $B$ have an odd number of incident edges. The introduction of a fictitious edge between them reduces the graph in canonical form. The augmented graph can now be covered by the path $A \rightarrow B \rightarrow E \rightarrow A$ which traverses exactly once each edge of the graph.

Through the use of the covering path, a query can be represented as a linear formula $\phi$ which chains object types and instances through spatial relations:

$\phi=t\left|t \stackrel{\omega}{\rightarrow} \phi^{\prime}\right| x \cdot \phi^{\prime} \mid \phi^{\prime}, x=y$

where: $t$ is an object type; $w$ is a 9-tuple of walkthroughs weights; and $x$ and $y$ are freeze-variables taken from an infinite support of object identifiers.

Semantics of a formula $\phi$ is defined by the satisfaction relation $\vDash_{\mu}$ which tells the degree of similarity by which an object within a description with a given assignment of object identifiers to freeze variables satisfies $\phi$. Given a formula $\phi$, a description $d=\langle O b j, W W$, Type $>$, an object $o b j \in O b j$, and an interpretation $\Gamma$ which assigns to each freeze variable an object $o b j \in O b j$, we say that under the interpretation $\Gamma, o b j$ is a starting point for a formula $\phi$ with similarity not lower than $\mu \in[0,1]$, and we write $<o b j, \Gamma>\models_{\mu} \phi$, in accordance with the following four inductive clauses.

- $<o b j, \Gamma>\vDash_{\mu} t$ iff Type $(o b j)=t$.

- $\left\langle o b j, \Gamma>\vDash_{\mu} t \stackrel{\omega}{\rightarrow} \phi \prime\right.$ iff there exists an object $o b j \prime \in O b j$ and three values of the similarity degree $\mu_{1}, \mu_{2}$ and $\mu_{3}$, such that $\mu \leq \sqrt[3]{\mu_{1} * \mu_{2} * \mu_{3}}$ and:

$$
\begin{aligned}
& <o b j, \Gamma>\vDash_{\mu_{1}} t ; \\
& \mathcal{S}\left(W W(<o b j, o b j \prime>, w) \geq \mu_{2} ;\right.
\end{aligned}
$$




$$
<o b j \prime, \Gamma>\vDash_{\mu_{3}} \phi \prime
$$

- $\left\langle o b j, \Gamma>\models_{\mu} x . \phi^{\prime}\right.$ iff $\Gamma(x)=-1$ and $\left\langle o b j, \Gamma \prime>\models_{\mu} \phi^{\prime}\right.$ where $\Gamma^{\prime}$ is the interpretation of freeze variables which assigns object $o b j$ to the rariable $x$ and agrees with $\Gamma$ for any other freeze variable different than $x$.

- $<o b j, \Gamma>\vDash_{\mu} \phi \prime, x=y \quad$ iff $\Gamma(x)=\Gamma(y)$ and $<o b j, \Gamma>\vDash_{\mu} \phi \prime$.

Referring again to the example of Fig.4, the querying semantics of the path $A \rightarrow B \rightarrow E \rightarrow A$ is represented by the sequential formula $\phi$ :

$\phi=x \cdot A \stackrel{\bar{w}(A B)}{\rightarrow} B \stackrel{\bar{w}_{\text {any }}}{\rightarrow} C \stackrel{\bar{w}(E A)}{\rightarrow} y \cdot A, x=y \quad$,

where $\bar{w}(A B), \bar{w}_{\text {any }}$, and $\bar{w}(E A)$ denote the walkthroughs associated with the edges of the graph of Fig.4.

Semantic clauses of linear formulae $\phi$ can be cast into a model checking algo-

rithm which operates through two subsequent stages performing a top-down syntactic decomposition of the formula and a bottom-up semantic labeling of the image description graph. In the first stage, formula $\phi$ is recursively decomposed in sub-formulae of decreasing length by applying the syntactic BNF structure of Eq.(9). For instance, the formula of Eq.(10) is decomposed as:

$$
\begin{array}{llll}
\phi=\phi_{1}, x=y & \phi_{1}=x \cdot \phi_{2} & \phi_{2}=A \stackrel{\bar{w}(A B)}{\rightarrow} \phi_{3} & \\
\phi_{3}=B \stackrel{\bar{w}_{\text {any }}}{\rightarrow} \phi_{4} & \phi_{4}=E \stackrel{\bar{w}(E A)}{\rightarrow} \phi_{5} & \phi_{5}=y \cdot \phi_{6} & \phi_{6}=A
\end{array}
$$

In the second stage, the nodes of the image graph are recursively labeled with the degree of similarity of sub-formulae that are satisfied in the node itself. The maximum degree of similarity obtained on any object for the main formula represents the degree of similarity between the image description and the querying sketch.

The labeling proceeds incrementally in a bottom-up manner starting with sub-formulae of minimum length. On the $n^{\text {th }}$ step, the labeling of formulae with length lower than $n$ is used to compute the labeling of the formulae with length $n+1$.

\section{DISCUSSION}

An original technique for modeling of spatial relationships between imaged objects has been introduced. The model, referred to as weighted walkthroughs, represents the mutual positioning of two objects as a 9-tuple of weights, each providing integral measure for the significance of one out of nine alternative directional relationships. By resorting to a property of compositionality, these 
integral weights can be derived by linear combination of a strict set of basic forms, thus circumventing the computational complexity of integral evaluation.

The use of multiple weights encompasses vague relationships between complex and near objects without requiring their replacement through centroids or minimum embedding rectangles. Besides, the use of quantitative weights enables metric evaluation of similarity supporting imprecise matching. Finally, integral derivation of weights supports analytical treatment and ensures continuity in the relation between spatial arrangements and their descriptions.

Weighted walkthroughs have been embedded within an original graphtheoretical framework supporting representation of spatial arrangements among multiple typed objects, each type allowing for multiple instances. A model checking approach permits quantitative evaluation of similarity among different spatial arrangements with corresponding types. This opens the way to the use of the entire framework as theoretical basis of engines for visual retrieval by contents supporting similarity matching between image descriptions and user's sketches.

Solution of trade-offs encountered along the design of the model has been tailored to support the development of a retrieval system for a database of images reproducing paintings of the italian renaissance period. Nevertheless, exploitation of the resulting framework can be spread to a large variety of applications requiring inexact management of spatial knowledge. In particular. the capability of the model-checking algorithm to support imprecise matching between similar but different spatial arrangement can be naturally extended to encompass metric evaluation of similarity between object types. This opens the way to the usage of the proposed framework in the representation and comparison of spatial arrangements between generic image segments, characterized by their high-level semantic type (as described in this paper) or by some low-level cohesion feature (such as color or texture distribution).

\section{REFERENCES}

[1] J.F.Allen, "Maintaining Knowledge about Temporal Intervals," Communications of the $A C M$, Vol.26, No.11, pp.832-843, Nov.1983.

[2] S.K.Chang, E.Jungert, T.Li, "Representation and Retrieval of Symbolic: Pictures Using Generalized 2D Strings," SPIE Proc. of Visual Communications and Image Processing IV, Vol.1199, pp.1360-1372, SPIE, 1989.

[3] S.K.Chang, Q.Y.Shi, C.W.Yan, "Iconic Indexing by 2-D Strings", IEEE Transactions on Pattern Analysis and Machine Intelligence. Vol.9, No.3, pp.413-427, July 1987.

[4] S.K.Chang, E.Jungert, "Pictorial Data Management Based upon the Theory of Symbolic Projections," Journal of Visual Languages and Computing, Vol.2, No.2, pp.195-215, June 1991. 
[5] A.Del Bimbo, E.Vicario, "Specification by-Example of Virtual Aycuts Behavior," IEEE Transactions on Visualization and Computer Graphics, Vol.1, No.4, Dec.1995.

[6] M.De Marsico, L.Cinque, S.Levialdi, "Indexing pictorial documents by their content: a survey of current techniques," Image and Vision Computing, Elsevier, Vol.15, Feb.1997, pp.119-141.

[7] M.J.Egenhofer, R. Franzosa, "On the Equivalence of Topological Relations," International Journal of Geographical Information Systems, Vol. 9, n. 2, 1992

[8] H.A.Eiselt, M.Gendreau, G.Laporte, "Arc Routing Problems, Part I: the Chinese Postman Problem," Operations Research, Vol.43, No.2, MarchApril 1995.

[9] C.Freksa, "Using Orientation Information for Qualitative Spatial Reasoning," Proc. of Int.Conf.on Theories and Methods of Spatio-Temporal Reasoning in Geographic Space, Pisa, Sept. 1992, Lecture Notes in Computer Science, Springer Verlag, 1992, pp.162-177.

[10] V.N.Gudivada, V.V.Raghavan, Special Issue on Content Based Image Retrieval Systems, Computer, Vol.28, No.9, 1995.

[11] V.N.Gudivada, V.V.Raghavan, "Design and Evaluation of Algorithms for Image retrieval by Spatial Similarity," ACM Trans. on Information Systems, Vol.13, No.2, April 1995.

[12] A.Gupta, R.Jain, "Visual Information Retrieval," Communications of the $A C M$, Vol.40, No.5, pp.70-79, May 1997.

[13] P.D.Holmes, E.Jungert, "Symbolic and Geometric Connectivity Graph Methods for Route Planning in Digitized Maps," IEEE Trans. on Pattern Analysis and Machine Intelligence, Vol. 14, n. 5, May 1992

[14] E.Jungert, "Qualitative Spatial Reasoning for Determination of Object Relations Using Symbolic Interval Projections," IEEE Int.Workshop on Visual Languages VL'93, pp.83-87.

[15] S.Lee, and F.Hsu, "Spatial Reasoning and Similarity Retrieval of Images using 2D C-strings Knowledge Representation," Pattern Recognition, 25(3):305-318, 1992.

[16] S.Y.Lee, M.C.Yang, and J.W.Cheng, "Signature file as spatial filter for iconic image database," Journal of Visual Languages and Computing, Vol.3, No. 4, pp.373-397, 1992. 